\title{
Investigation of the possibility of increasing the sensitivity of a microwave radiometer
}

\author{
Alexander Luchinin ${ }^{1, *}$ and Ivan Malygin ${ }^{1}$ \\ ${ }^{1}$ URFU, Department of Radio Electronics and Communications, 620002 Yekaterinburg, Russia
}

\begin{abstract}
The results of a sensitivity study of various types of quadratic detectors for a microwave radiometer are presented, both with and without a low-noise amplifier. A low-noise amplifier with a gain of $60 \mathrm{~dB}$ and a bandwidth of $1 \mathrm{GHz}$ at a central frequency of $4 \mathrm{GHz}$ was developed and studied.
\end{abstract}

\section{Introduction}

The measurement of microwave energy using radiometers has been known for over 80 years [1-3]. Microwave radiometers are widely used in medical, astrophysical and other scientific fields.

In medicine there is a need to build multi-channel radiometers or, similarly, to use a large number of radiometers. Theoretically, the modern element base allows building of relatively cheap radiometers using undeveloped technological base. The present work estimates this possibility with an attempt to construct a radiometer "at home".

The crucial characteristic ensuring the measurements accuracy using a radiometer is the threshold of sensitivity. Regardless of the method of radiometer constructing, the threshold of sensitivity is determined by the level of intrinsic noises of the microwave amplifier and subsequent elements of the analog signal processing: quadratic detector, low-frequency amplifier.

It is known that the threshold of sensitivity of radiometers increases correspondingly to the ratio of the microwave amplifier bandpass and low-frequency path [1]:

$$
\Delta T=T_{N} \cdot \sqrt{\frac{\Delta f_{L F}}{\Delta f_{V H F}}}
$$

where $\Delta T$ is the sensitivity threshold of the radiometer, presented in degrees Kelvin; $T_{N}$ noise temperature of the analog microwave circuit; $\Delta f_{V H F}$ - bandwidth of the microwave amplifier; $\Delta f_{L F}$ is the amplifier bandwidth after the quadratic detector.

During the process, a broadband low-noise microwave amplifier with a gain of $60 \mathrm{~dB}$ was designed and three types of detectors were investigated: detectors on the Schottky diodes HSMS-2862, a logarithmic detector on an AD8318 chip and a detector on an IAM91563 chip.

* Corresponding author: r3031as@mail.ru 


\section{Objects of research and experimental technique}

In accordance with the expression (1), to obtain a high sensitivity of the radiometer, it is necessary to expand the microwave amplifier bandpass and provide the lowest possible noise temperature of the microwave path - the amplifier and the subsequent quadratic detector.

In this work, we investigated the possibility to construct a radiometer operating in the frequency range 3-4 GHz. To build a low-noise broadband amplifier within this range, modern catalogs provide rather wide selection of amplifier circuits with the noise of less than $1-2 \mathrm{~dB}$.

A more difficult task is to build a low-noise, highly sensitive quadratic detector.

Existing circuitry solutions of radiometers frequently offer to build a detector according to a simple scheme - a diode detector in series. High sensitivity of the radiometer is ensured by a large amplifier coefficient - more than $60 \mathrm{~dB}$. The construction of such amplifier requires careful design ensuring stable operation without self-excitation.

The present work shows experiments to construct a broadband microwave amplifier operating within the range from 3.3 to $4.3 \mathrm{GHz}$ with a gain of more than $60 \mathrm{~dB}$. The lownoise amplifier is created according to a three-stage scheme on MGA-86576 microcircuits. There are bandpass filters designed for a $1 \mathrm{GHz}$ bandwidth at the centre frequency of 3.8 $\mathrm{GHz}$ between the cascades. The frequency response of the amplifier is shown in Fig. 1.

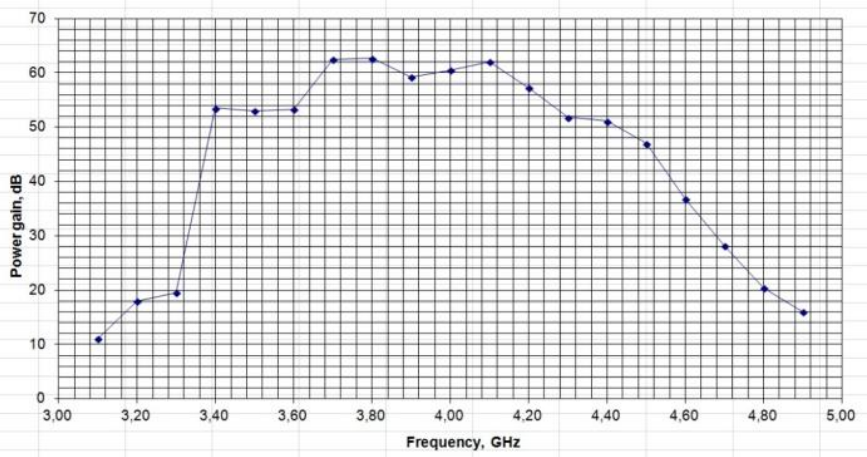

Fig. 1. The characteristic of low-noise microwave amplifier. Noise Band $0.8 \mathrm{GHz}$.

In order to simplify the amplifier - to reduce the gain, there were conducted experiments to build various versions of quadratic detectors. The highest sensitivity was achieved in a diode detector with voltage doubling and the offset of the operating point through the direct current. The detector is built on Schottky diodes HSMS-2862. The characteristic is given in Fig. 2. The characteristic is measured at $4 \mathrm{GHz}$ centre frequency with pulse modulation of the input signal with $3 \mathrm{kHz}$ frequency, the duty cycle 2 .

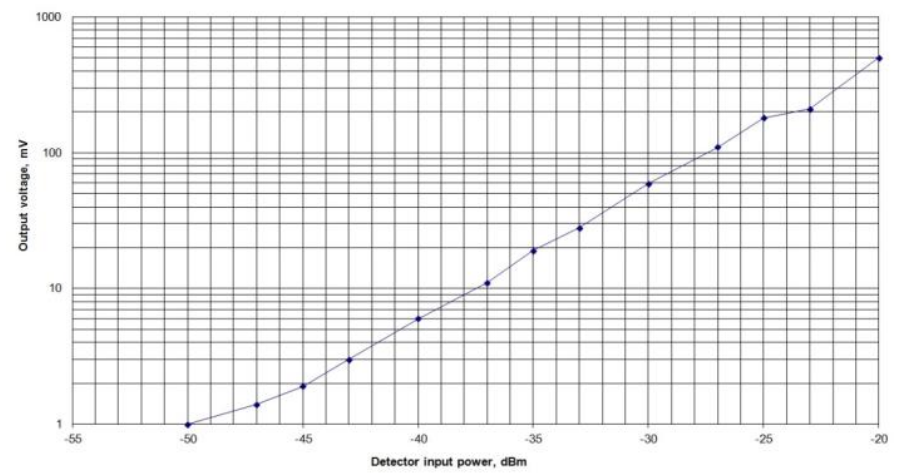

Fig. 2. HSMS-2862 Schottky Diode Detector Feature. 


\section{Method for measuring the noise level of main elements of the radiometer}

The threshold of sensitivity measurement (assessment) of the radiometer was conducted through a mode that implements a modulation method of achieving high sensitivity. The main elements determining the threshold of sensitivity of the radiometer are the microwave amplifier, circulator and switch, which are switched on at the input of the amplifier. Therefore, two measurements were performed: the noise level of a microwave amplifier with a quadratic detector; the noise level of a microwave amplifier with a circulator and a switch switched on at the input.

The block diagram of the installation of noise measurement of these elements is shown in Fig. 3.

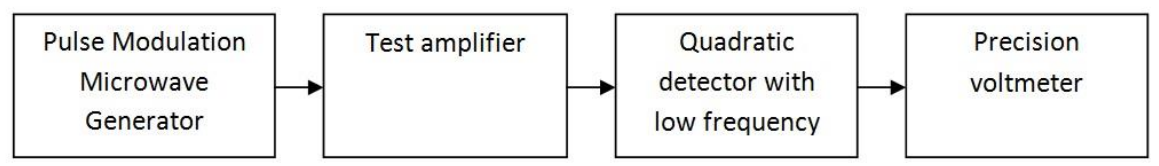

Fig. 3. Block diagram of the installation of the noise level measurement of radiometer elements.

A signal from a microwave generator (SMA 100, Rohde \& Schwartz) with pulse modulation by a rectangular low-frequency signal is given to the input of studied elements, the duty cycle is 2 .

The output voltage is measured with an AC voltmeter V3 - 38 .

A quadratic voltmeter is connected to the output of the microwave amplifier followed by a low-frequency amplifier. The ULF bandwidth is from $100 \mathrm{~Hz}$ to $5 \mathrm{kHz}$. The gain is 3000 .

The voltmeter is calibrated within current values of sinusoidal voltage. Accounting some errors in our measurements, it is considered that the measurement result corresponds to the actual value of the rectangular voltage at the output of the quadratic detector (turned on at the output of the microwave amplifier).

The procedure of noise measurement of a microwave amplifier includes three stages:

1) noise level is measured at the output of the measuring installation (Fig. 3) with the switched off microwave generator;

2) the microwave generator (with pulse modulation) is switched on and the power level of its carrier is selected so that the voltmeter readings increase by $3 \mathrm{~dB}$;

3 ) the readings of the power scale of the microwave generator carrier are read.

The measurement results in accordance with paragraph (2) are shown in Fig. 4. The figure shows the results obtained at different carrier frequencies of the input microwave oscillations.

A significant frequency dependence of the intrinsic noise level of the microwave amplifier - quadratic detector with low frequency amplifier is observed. The frequency dependence of the noise level almost repeats the frequency response of the microwave amplifier. This behavior of the frequency dependence of the noise level shows that despite the high gain of the microwave amplifier (more than $60 \mathrm{~dB}$ ) the level of intrinsic noise of the microwave amplifier-detector system is determined by the noise of the amplitude detector. Thus, to increase the sensitivity of the radiometer, the gain of the microwave amplifier should be further increased.

Based on the measurement result, Fig. 4, the noise level of the microwave amplifierdetector system is calculated. 


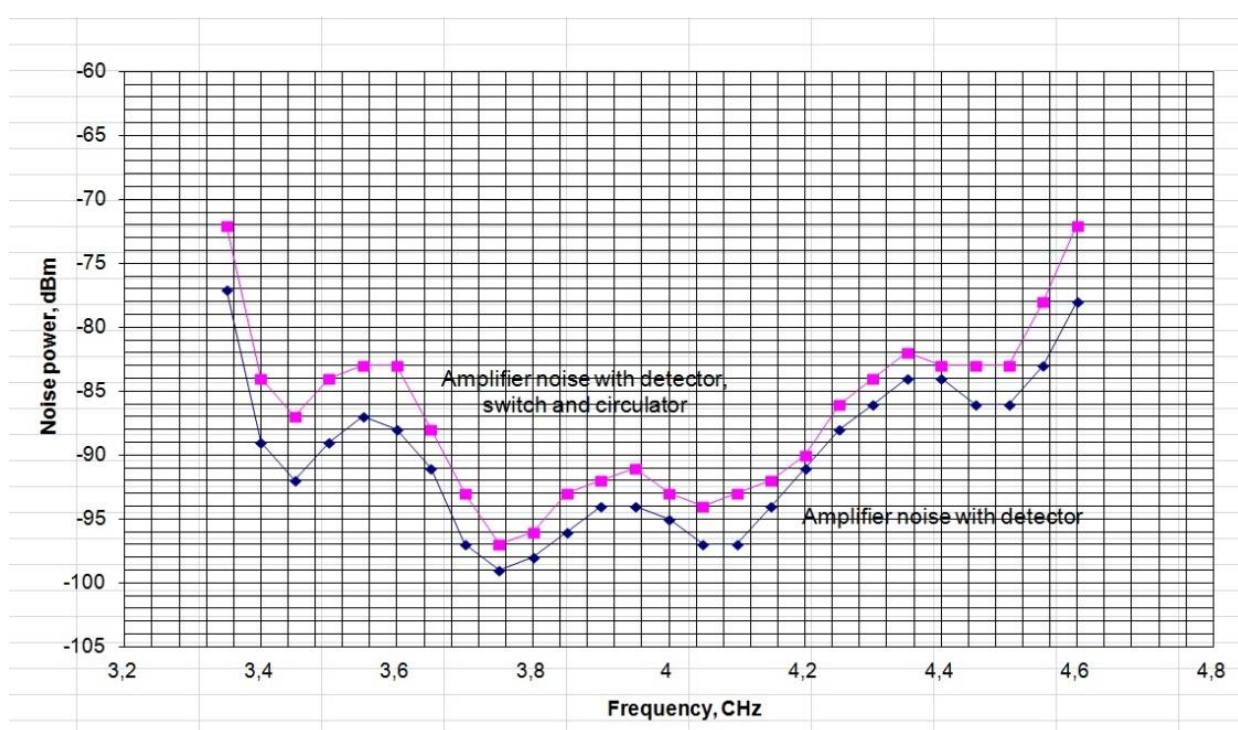

Fig. 4. The result of measuring the noise level of radiometer elements.

The minimum value of the carrier power shown in Fig. 2, is $-99 \mathrm{dBm}$. The deviation of the amplitude of the input harmonic microwave oscillation given to the amplifier input from the average value is half the amplitude $(-6 \mathrm{~dB})$, modulation coefficient $\mathrm{M}=100 \%$. Thus, the effective value of the input signal (determined by modulation) is $-105 \mathrm{~dB}$.

This value of the input signal power is equal to the noise power of the amplifier (reduced to the input of the amplifier). Compare the obtained power with the expression for the thermal noise power [8]:

$$
P_{N}=k T \Delta F
$$

where $k$ is the Boltzmann constant, $\Delta F$ is the bandwidth of the microwave amplifier (equal to $0.8 \mathrm{GHz}), T$ is the noise temperature.

Determine the value of the noise temperature: $\Delta T=2.86^{\circ} \mathrm{K}$.

The use of the modulation method to increase the radiometer threshold of sensitivity allows reducing the noise temperature of the input part of the radiometer [1].

In accordance with (2), the noise temperature of the studied microwave amplifier with a detector is $T_{S}=1144^{\circ} \mathrm{K}$. The system noise figure at a standard temperature of $+20^{\circ} \mathrm{C}$ $\left(293^{\circ} \mathrm{K}\right)$ is $F=4.9(6.9 \mathrm{~dB})$.

Turning on the input of the microwave amplifier of the switch and the circulator, the noise figure increases by $2 \mathrm{~dB}$.

The noise temperature of system "microwave amplifier-circulator-switch" using the modulation method of measurement increases to $\Delta T=4.54^{\circ} \mathrm{K}$.

\section{Conclusion}

During the process, a low-noise amplifier and three types of quadratic detectors were created and investigated. The detector based on the IAM-91563 microcircuit has the worst characteristic from the studied samples - at the operating frequency its sensitivity does not exceed $-18 \mathrm{dBm}$. Logarithmic detector based on AD8318 chip has the best performance. During the measurements, the sensitivity indicated in the documentation was confirmed no less than $60 \mathrm{dBm}$. During the experiment, the characteristics of the detector on Schottky diodes HSMS-2862 were confirmed no less than $55 \mathrm{dBm}$. The diode detector was turned on according to the scheme with doubling and bias. 


\section{References}

1. V.S. Kublanov, M.V. Babich, T.S. Petrenko, Biomedical Engineering, v. 52, 9 (2018)

2. P.G. Tsybulev, M.V. Dugin, A.B. Berlin, N.A. Nizhelskij, D.V. Kratov, R.Y. Udovitskiy, Astrophysical Bulletin, v. 69, 240 (2014)

3. V.K. Volosyuk, V.V. Pavlikov, Radioelectronics and Communications Systems, v. 55, $433(2012)$

4. A.K. Blinov, A.M. Korolev, V.V. Myshenko, S.A. Peskovatskii, V.I. Podyachii, V.M. Shulga, International Conference on Millimeter Wave and Far-Infrared Technology Proceedings, 12646 (1989)

5. J. E. Ohlson, Radio Science, v. 6(3), 341 (1971)

6. E.V. Fedoseeva, G.G. Shchukin, D.M. Karavaev, CriMiCo 2014 - 2014 24th International Crimean Conference Microwave and Telecommunication Technology, Conference Proceedings, 6959781, 1105 (2014)

7. A.V. Filatov, N.A. Karataeva, V.D. Dmitriev, Measurement Techniques, v. 50, 337 (2007)

8. M.J. Buckingham, Noise in electronic devices and systems (John Wiley\&Sons, New York, 1983) 